\title{
Strong laws of large numbers for general random variables in sublinear expectation spaces
}

\author{
Weihuan Huang ${ }^{1}$ and Panyu $\mathrm{Wu}^{1 *}$ (D)
}

\section{"Correspondence:}

wupanyu@sdu.edu.cn

'Zhongtai Institute for Financial Studies, Shandong University, Jinan, China

\begin{abstract}
In this paper, we obtain the equivalent relations between Kolmogorov maximal inequality and Hájek-Rényi maximal inequality both in moment and capacity types in sublinear expectation spaces. Based on these, we establish several strong laws of large numbers for general random variables and obtain the growth rate of the partial sums. In a first application, a strong law of large numbers for negatively dependent random variables is obtained. In a second application, we consider the normalizing sequence $\{\log n\}_{n \geq 1}$ and get some special limit properties in sublinear expectation spaces.
\end{abstract}

Keywords: Maximal inequality; Sublinear expectation; Strong law of large numbers

\section{Introduction and notations}

The general framework of the sublinear expectation space was introduced by Peng [13] and it is a natural extension of the classical linear expectation space with the linear property being replaced by the subadditivity and positive homogeneity. This simple generalization provides a very flexible framework to model uncertainty problems in statistics or finance. Peng [14] introduced the independent and identically distributed random variables, G-normal distribution and obtained the weak law of large numbers and the central limit theorem in sublinear expectation spaces. Since then, many authors began to investigate the limit properties, especially the law of large numbers in sublinear expectation spaces. For instance, Chen, $\mathrm{Wu}$ and $\mathrm{Li}$ [5] gave a strong law of large numbers for non-negative product independent random variables; Chen [2] obtained the Kolmogorov strong law of large numbers for independent and identically distributed random variables; Zhang [21] gave the necessary and sufficient conditions of Kolmogorov strong law of larger numbers holding for independent and identically distributed random variables under a continuous sublinear expectation; Zhang [22] obtained a strong law of large numbers for a sequence of extended independent random variables; Chen, $\mathrm{Hu}$ and Zong [3] gave several strong laws of large numbers under some moment conditions with respect to the partial sum and some conditions similar to Petrov's; Chen, Huang and Wu [4] established an extension of the strong law of large numbers under exponential independence. There are also many results on law of large numbers under different non-additive probability framework. The reader can see $[1,6,12,18]$ and the references therein.

(c) The Author(s) 2019. This article is distributed under the terms of the Creative Commons Attribution 4.0 International License (http://creativecommons.org/licenses/by/4.0/), which permits unrestricted use, distribution, and reproduction in any medium, provided you give appropriate credit to the original author(s) and the source, provide a link to the Creative Commons license, and indicate if changes were made. 
However, none of these papers investigate the growth rate of the partial sums. The main purpose of this paper is to explore the growth rate of the partial sums and investigate the strong law of large numbers for general random variables in sublinear expectation spaces.

In classical probability or expectation space, Fazekas and Klesov [9] gave a general approach of obtaining the strong law of large numbers for sequences of random variables by using a Hájek-Rényi type maximal inequality. This general approach made no restriction on the dependence structure of random variables. Under the same conditions as those in Fazekas and Klesov [9], $\mathrm{Hu}$ and $\mathrm{Hu}$ [10] obtained the growth rate for the sums of random variables. Sung, Hu and Volodin [17] improved these results and applied this approach to the weak law of large numbers for tail series. Tómács and Líbor [20] used the probability Hájek-Rényi type maximal inequality to prove the strong law of large numbers. More results and applications of this approach in classical probability space can be found in Fazekas [8] and the references therein.

In this paper, we will extend the approach used by Fazekas and Klesov [9] and $\mathrm{Hu}$ and $\mathrm{Hu}$ [10] to sublinear expectation spaces. In Sect. 2, we firstly show the equivalent relations between Kolmogorov maximal inequality and Hájek-Rényi maximal inequality both in the moment and capacity types in sublinear expectation spaces. Based on these, we establish several strong laws of large numbers for general random variables in sublinear expectation spaces and obtain the growth rate of the partial sums in Sect. 3. As an application, a strong law of large numbers for negatively dependent random variables is obtained since the Kolmogorov type maximal inequality for negatively dependent random variables has been proved in Sect. 2. In Sect. 4, we give another application. We consider the normalizing sequence $\{\log n\}_{n \geq 1}$ and get some limit properties in sublinear expectation spaces.

In the remainder of this section, we give some notations in sublinear expectation spaces. We use the framework and notations in Peng [14] and [15]. Let $(\Omega, \mathcal{F})$ be a given measurable space and $\mathscr{H}$ be a linear space of real measurable functions defined on $(\Omega, \mathcal{F})$ such that if $X_{1}, \ldots, X_{n} \in \mathscr{H}$ then $\psi\left(X_{1}, \ldots, X_{n}\right) \in \mathscr{H}$, for each $\psi \in C_{l, \text { Lip }}\left(\mathbb{R}^{n}\right)$, where $C_{l, \text { Lip }}\left(\mathbb{R}^{n}\right)$ denotes the linear space of functions $\psi$ satisfying

$$
|\psi(y)-\psi(z)| \leq C\left(1+|y|^{m}+|z|^{m}\right)|y-z|
$$

for all $y, z \in \mathbb{R}^{n}$, for some $C>0, m \in \mathbb{N}$ depending on $\psi$.

Here and in the sequel, $\mathbb{N}$ denotes the set of all non-negative integers and $\mathbb{N}^{*}$ denotes the set of all positive integers. In general, $C_{l, \text { Lip }}\left(\mathbb{R}^{n}\right)$ can be replaced by $C_{b, \text { Lip }}\left(\mathbb{R}^{n}\right)$, which is the space of all bounded and Lipschitz continuous functions on $\mathbb{R}^{n}$. The space $\mathscr{H}$ is considered as a space of random variables.

Definition 1.1 A sublinear expectation $\mathbb{E}$ on $\mathscr{H}$ is a functional $\mathbb{E}: \mathscr{H} \rightarrow \mathbb{R} \cup\{-\infty, \infty\}$ satisfying the following properties: for all $X, Y \in \mathscr{H}$, we have

(a) monotonicity: $\mathbb{E}[X] \geq \mathbb{E}[Y]$, if $X \geq Y$;

(b) constant preserving: $\mathbb{E}[c]=c$, for $c \in \mathbb{R}$;

(c) subadditivity: $\mathbb{E}[X+Y] \leq \mathbb{E}[X]+\mathbb{E}[Y]$ whenever $\mathbb{E}[X]+\mathbb{E}[Y]$ is not of the form $\infty-\infty$ or $-\infty+\infty$

(d) positive homogeneity: $\mathbb{E}[\lambda X]=\lambda \mathbb{E}[X]$, for $\lambda \geq 0$. 
The triple $(\Omega, \mathscr{H}, \mathbb{E})$ is called a sublinear expectation space. Given a sublinear expectation $\mathbb{E}$, let us denote the conjugate expectation $\mathcal{E}$ of $\mathbb{E}$ by

$$
\mathcal{E}[X]:=-\mathbb{E}[-X], \quad \text { for all } X \in \mathscr{H} .
$$

Lemma 1.2 (Lemma 2.4 in Peng [14]) A functional $\mathbb{E}$ defined on $(\Omega, \mathscr{H})$ is a sublinear expectation if and only if, there exists a family of linear expectations (finite additive) $\left\{E_{\theta}\right.$ : $\theta \in \Theta\}$ such that

$$
\mathbb{E}[X]=\max _{\theta \in \Theta} E_{\theta}[X], \quad \text { for all } X \in \mathscr{H} .
$$

Obviously, for all $X \in \mathscr{H}, \mathcal{E}[X] \leq \mathbb{E}[X]$. Furthermore, $(\mathbb{E}, \mathcal{E})$ can generate a pair $(V, v)$ of capacities by

$$
V(A):=\sup _{\theta \in \Theta} E_{\theta}\left[I_{A}\right], \quad v(A):=1-V\left(A^{c}\right), \quad \text { for all } A \in \mathcal{F},
$$

where $\left\{E_{\theta}: \theta \in \Theta\right\}$ is the family of linear expectations in Lemma 1.2 and $A^{c}$ is the complement set of $A$. It is easy to check that $V$ is subadditive, monotonic and

$$
\mathbb{E}[X] \leq V(A) \leq \mathbb{E}[Y], \quad \mathcal{E}[X] \leq v(A) \leq \mathcal{E}[Y], \quad \text { if } X \leq I_{A} \leq Y, X, Y \in \mathscr{H} .
$$

Moreover, if $I_{A} \in \mathscr{H}$, then $V(A)=\mathbb{E}\left[I_{A}\right]$ and $v(A)=\mathcal{E}\left[I_{A}\right]$.

Remark 1.3 Zhang [21] introduced another way to define the capacities generated by $(\mathbb{E}, \mathcal{E})$, that is,

$$
\bar{V}(A):=\inf \left\{\mathbb{E}[X]: I_{A} \leq X, X \in \mathscr{H}\right\}, \quad \bar{v}(A):=1-V\left(A^{c}\right), \quad \text { for all } A \in \mathcal{F} .
$$

Under this definition, $\bar{V}$ is also subadditive, monotonic and $(\bar{V}, \bar{v})$ satisfy (1). The relation of these two pair of capacities satisfy $\bar{v}(A) \leq v(A) \leq V(A) \leq \bar{V}(A)$, for all $A \in \mathcal{F}$. More properties about $(\bar{V}, \bar{v})$ can be found in Zhang [21]. It is easy to see that Theorem 2.2, Proposition 2.5 and Theorem 2.7 in this paper still hold when $(V, v)$ is replaced by $(\bar{V}, \bar{v})$, respectively.

\section{Definition 1.4}

(i) In a sublinear expectation space $(\Omega, \mathscr{H}, \mathbb{E})$, a $n$-dimensional random vector $\mathbf{Y}$ is said to be independent on another $m$-dimensional random vector $\mathbf{X}$ under $\mathbb{E}$ if for each test function $\psi \in C_{l, \text { Lip }}\left(\mathbb{R}^{m+n}\right)$ we have

$$
\mathbb{E}[\psi(\mathbf{X}, \mathbf{Y})]=\mathbb{E}\left[\left.\mathbb{E}[\psi(\mathbf{x}, \mathbf{Y})]\right|_{\mathbf{x}=\mathbf{X}}\right],
$$

whenever $\bar{\psi}(\mathbf{x}):=\mathbb{E}[|\psi(\mathbf{x}, \mathbf{Y})|]<\infty$ for all $\mathbf{x}$ and $\mathbb{E}[|\bar{\psi}(\mathbf{X})|]<\infty$.

(ii) A sequence of random variables $\left\{X_{n}\right\}_{n \geq 1}$ is said to be independent, if $X_{n+1}$ is independent on $\left(X_{1}, \ldots, X_{n}\right)$ for each $n \geq 1$. 
Definition 1.5 (Definition 1.5 in Zhang [21]) In a sublinear expectation space $(\Omega, \mathscr{H}, \mathbb{E})$, a $n$-dimensional random vector $\mathbf{Y}$ is said to be negatively dependent on another $m$ dimensional random vector $\mathbf{X}$ under $\mathbb{E}$, if for each pair of test function $\psi_{1} \in C_{l, \text { Lip }}\left(\mathbb{R}^{m}\right)$ and $\psi_{2} \in C_{l, \text { Lip }}\left(\mathbb{R}^{n}\right)$, we have $\mathbb{E}\left[\psi_{1}(\mathbf{X}) \psi_{2}(\mathbf{Y})\right] \leq \mathbb{E}\left[\psi_{1}(\mathbf{X})\right] \mathbb{E}\left[\psi_{2}(\mathbf{Y})\right]$, whenever $\psi_{1}(\mathbf{X}) \geq 0$ and $\mathbb{E}\left[\psi_{2}(\mathbf{Y})\right] \geq 0, \mathbb{E}\left[\left|\psi_{1}(\mathbf{X}) \psi_{2}(\mathbf{Y})\right|\right]<\infty, \mathbb{E}\left[\left|\psi_{1}(\mathbf{X})\right|\right]<\infty, \mathbb{E}\left[\left|\psi_{2}(\mathbf{Y})\right|\right]<\infty$ and either $\psi_{1}$ and $\psi_{2}$ are coordinatewise nondecreasing or $\psi_{1}$ and $\psi_{2}$ are coordinatewise non-increasing.

\section{Kolmogorov type and Hájek-Rényi type maximal inequalities}

For convenience, let $\max _{i \in A} a_{i}=0$ and $\sum_{i \in A} a_{i}=0$ if $A=\emptyset$.

Let $\left\{X_{i}\right\}_{i \geq 1}$ denote a sequence of random variables in the sublinear expectation space $(\Omega, \mathscr{H}, \mathbb{E})$ and the partial sums of random variables be $S_{n}=\sum_{i=1}^{n} X_{i}$ for all $n \in \mathbb{N}^{*}$ and $S_{0}=0$. The constant $c$ may be different in different places.

Theorem 2.1 Let $\left\{\alpha_{l}\right\}_{l=1}^{n}$ be a sequence of non-negative numbers and $r>0$. Then the following two statements are equivalent:

(i) there exists a constant $c>0$, such that, for any $k=1, \ldots, n$,

$$
\mathbb{E}\left[\max _{1 \leq l \leq k}\left|S_{l}\right|^{r}\right] \leq c \sum_{l=1}^{k} \alpha_{l}
$$

(ii) there exists a constant $c>0$, such that, for any nondecreasing positive numbers

$\left\{\beta_{l}\right\}_{l=1}^{n}$ and any $k=1, \ldots, n$,

$$
\mathbb{E}\left[\max _{1 \leq l \leq k}\left|\frac{S_{l}}{\beta_{l}}\right|^{r}\right] \leq 4 c \sum_{l=1}^{k} \frac{\alpha_{l}}{\beta_{l}^{r}} .
$$

Proof In (ii), for $l=1, \ldots, n$, let $\beta_{l} \equiv 1$, then we can get (i) from (ii). Now we turn to the proof of (i) $\Rightarrow$ (ii). The proof is based on the idea of the proof of Theorem 1.1 by Fazekas and Klesov [9].

Fix any nondecreasing positive numbers $\left\{\beta_{l}\right\}_{l=1}^{n}$. Without loss of generality we can assume $\beta_{1}=1$. For any fixed $k \in\{1, \ldots, n\}$, define the following notations:

$$
\begin{aligned}
& A_{i}=\left\{l: 1 \leq l \leq k \text { and } 2^{i} \leq \beta_{l}^{r}<2^{i+1}\right\}, \quad i \in \mathbb{N}, \\
& I=\max \left\{i: A_{i} \neq \emptyset\right\}, \\
& m(i)=\left\{\begin{array}{ll}
\max \left\{l: l \in A_{i}\right\}, & \text { if } A_{i} \neq \emptyset, \\
m(i-1), & \text { if } A_{i}=\emptyset,
\end{array} \quad i \in \mathbb{N}, \quad \text { and } \quad m(-1)=0 .\right.
\end{aligned}
$$

It is easy to see that $I<\infty, m(0) \geq 1$ and $\{m(i)\}_{i=-1}^{\infty}$ is nondecreasing. Then, by the subadditivity of $\mathbb{E}$ and inequality (2), we have

$$
\begin{aligned}
\mathbb{E}\left[\max _{1 \leq l \leq k}\left|\frac{S_{l}}{\beta_{l}}\right|^{r}\right] & \leq \sum_{i=0}^{I} \mathbb{E}\left[\max _{l \in A_{i}}\left|\frac{S_{l}}{\beta_{l}}\right|^{r}\right] \leq \sum_{i=0}^{I} 2^{-i} \mathbb{E}\left[\max _{l \in A_{i}}\left|S_{l}\right|^{r}\right] \\
& \leq \sum_{i=0}^{I} 2^{-i} \mathbb{E}\left[\max _{l \leq m(i)}\left|S_{l}\right|^{r}\right] \leq \sum_{i=0}^{I} 2^{-i} c \sum_{l=1}^{m(i)} \alpha_{l}
\end{aligned}
$$




$$
\begin{aligned}
& \leq c \sum_{i=0}^{I} 2^{-i} \sum_{j=0}^{i} \sum_{l=m(j-1)+1}^{m(j)} \alpha_{l}=c \sum_{j=0}^{I} \sum_{l=m(j-1)+1}^{m(j)} \alpha_{l} \sum_{i=j}^{I} 2^{-i} \\
& \leq 2 c \sum_{j=0}^{I} 2^{-j} \sum_{l=m(j-1)+1}^{m(j)} \alpha_{l}=2 c \sum_{j=0}^{I} 2^{-j} \sum_{l=m(j-1)+1}^{m(j)} \frac{\alpha_{l}}{\beta_{l}^{r}} \beta_{l}^{r} \\
& \leq 4 c \sum_{j=0}^{I} \sum_{l=m(j-1)+1}^{m(j)} \frac{\alpha_{l}}{\beta_{l}^{r}}=4 c \sum_{l=1}^{k} \frac{\alpha_{l}}{\beta_{l}^{r}} .
\end{aligned}
$$

Therefore, the proof of this theorem is completed.

Theorem 2.2 Let $\left\{\alpha_{l}\right\}_{l=1}^{n}$ be a sequence of non-negative numbers and $r>0$. Then the following two statements are equivalent:

(i) there exists a constant $c>0$, such that, for any $k=1, \ldots, n$, any $\epsilon>0$,

$$
V\left(\max _{1 \leq l \leq k}\left|S_{l}\right| \geq \epsilon\right) \leq c \epsilon^{-r} \sum_{l=1}^{k} \alpha_{l} ;
$$

(ii) there exists a constant $c>0$, such that, for any nondecreasing positive numbers $\left\{\beta_{l}\right\}_{l=1}^{n}$ and any $k=1, \ldots, n$, any $\epsilon>0$,

$$
V\left(\max _{1 \leq l \leq k}\left|\frac{S_{l}}{\beta_{l}}\right| \geq \epsilon\right) \leq 4 c \epsilon^{-r} \sum_{l=1}^{k} \frac{\alpha_{l}}{\beta_{l}^{r}} .
$$

Proof In (ii), for $l=1, \ldots, n$, let $\beta_{l} \equiv 1$, then we can get (i) from (ii). Now we focus on the proof of (i) $\Rightarrow$ (ii).

Fix any nondecreasing positive numbers $\left\{\beta_{l}\right\}_{l=1}^{n}$ and fix any $k \in\{1, \ldots, n\}$. Without loss of generality we can assume $\beta_{1}=1$. We use the same notations $A_{i}, I$ and $m(i)$ defined in the proof of Theorem 2.1. Then, by the subadditivity and monotonicity of $V$ and inequality (4), we have

$$
\begin{aligned}
V\left(\max _{1 \leq l \leq k}\left|\frac{S_{l}}{\beta_{l}}\right| \geq \epsilon\right) & \leq \sum_{i=0}^{I} V\left(\max _{l \in A_{i}}\left|\frac{S_{l}}{\beta_{l}}\right| \geq \epsilon\right) \\
& \leq \sum_{i=0}^{I} V\left(\max _{l \in A_{i}}\left|S_{l}\right| \geq \epsilon 2^{i / r}\right) \\
& \leq \sum_{i=0}^{I} V\left(\max _{l \leq m(i)}\left|S_{l}\right| \geq \epsilon 2^{i / r}\right) \\
& \leq \sum_{i=0}^{I} c \epsilon^{-r} 2^{-i} \sum_{l=1}^{m(i)} \alpha_{l} .
\end{aligned}
$$

Through the proof of Theorem 2.1, we have

$$
\sum_{i=0}^{I} c \epsilon^{-r} 2^{-i} \sum_{l=1}^{m(i)} \alpha_{l} \leq 4 c \epsilon^{-r} \sum_{l=1}^{k} \frac{\alpha_{l}}{\beta_{l}^{r}} .
$$

Hence, the proof of this theorem is completed. 
Remark 2.3 Inequalities (2) and (4) are Kolmogorov maximal inequalities in the moment and capacity types, respectively. As well, inequalities (3) and (5) are Hájek-Rényi maximal inequalities in the moment and capacity types, respectively.

At the end of this section, we give two Kolmogorov type maximal inequalities for negatively dependent random variables in sublinear expectation spaces. Before we do this, we prove two propositions which will be used in the proof of Kolmogorov type maximal inequality.

Proposition 2.4 Let $\left\{f_{i}\right\}_{i=1}^{n_{1}} \subset C_{l, \mathrm{Lip}}\left(\mathbb{R}^{n}\right)$ and $\left\{g_{i}\right\}_{i=1}^{m_{1}} \subset C_{l, \mathrm{Lip}}\left(\mathbb{R}^{m}\right)$ be coordinatewise nondecreasing or coordinatewise non-increasing functions. If n-dimensional random vector $\mathbf{Y}$ is negatively dependent on m-dimensional random vector $\mathbf{X}$ under $\mathbb{E}$, then $\left(f_{1}(\mathbf{Y}), \ldots, f_{n_{1}}(\mathbf{Y})\right)$ is negatively dependent on $\left(g_{1}(\mathbf{X}), \ldots, g_{m_{1}}(\mathbf{X})\right)$. In particular, $-\mathbf{Y}$ is negatively dependent on $-\mathbf{X}$.

Proof For any test functions $\psi_{1} \in C_{l, \text { Lip }}\left(\mathbb{R}^{m_{1}}\right)$ and $\psi_{2} \in C_{l, \text { Lip }}\left(\mathbb{R}^{n_{1}}\right)$ such that $\psi_{1}\left(g_{1}(\mathbf{X}), \ldots\right.$, $\left.g_{m_{1}}(\mathbf{X})\right) \geq 0$ and $\mathbb{E}\left[\psi_{2}\left(f_{1}(\mathbf{Y}), \ldots, f_{n_{1}}(\mathbf{Y})\right)\right] \geq 0$,

$$
\mathbb{E}\left[\left|\psi_{1}\left(g_{1}(\mathbf{X}), \ldots, g_{m_{1}}(\mathbf{X})\right) \psi_{2}\left(f_{1}(\mathbf{Y}), \ldots, f_{n_{1}}(\mathbf{Y})\right)\right|\right]<\infty
$$

$\mathbb{E}\left[\left|\psi_{1}\left(g_{1}(\mathbf{X}), \ldots, g_{m_{1}}(\mathbf{X})\right)\right|\right]<\infty, \mathbb{E}\left[\left|\psi_{2}\left(f_{1}(\mathbf{Y}), \ldots, f_{n_{1}}(\mathbf{Y})\right)\right|\right]<\infty$ and either $\psi_{1}$ and $\psi_{2}$ are coordinatewise nondecreasing or $\psi_{1}$ and $\psi_{2}$ are coordinatewise non-increasing, define $\tilde{\psi}_{1}(\cdot)=\psi_{1}\left(g_{1}(\cdot), \ldots, g_{m_{1}}(\cdot)\right)$ and $\tilde{\psi}_{2}(\cdot)=\psi_{1}\left(f_{1}(\cdot), \ldots, f_{n_{1}}(\cdot)\right)$. Then $\tilde{\psi}_{1} \in C_{l, \text { Lip }}\left(\mathbb{R}^{m}\right)$ and $\tilde{\psi}_{2} \in C_{l, \text { Lip }}\left(\mathbb{R}^{n}\right)$ are coordinatewise nondecreasing or $\tilde{\psi}_{1}$ and $\tilde{\psi}_{2}$ are coordinatewise nonincreasing. Meanwhile $\tilde{\psi}_{1}(\mathbf{X}) \geq 0$ and $\mathbb{E}\left[\tilde{\psi}_{2}(\mathbf{Y})\right] \geq 0, \mathbb{E}\left[\left|\tilde{\psi}_{1}(\mathbf{X}) \tilde{\psi}_{2}(\mathbf{Y})\right|\right]<\infty, \mathbb{E}\left[\left|\tilde{\psi}_{1}(\mathbf{X})\right|\right]<$ $\infty, \mathbb{E}\left[\left|\tilde{\psi}_{2}(\mathbf{Y})\right|\right]<\infty$. Therefore, by the definition of negatively dependence, we have

$$
\begin{aligned}
\mathbb{E} & {\left[\psi_{1}\left(g_{1}(\mathbf{X}), \ldots, g_{m_{1}}(\mathbf{X})\right) \psi_{2}\left(f_{1}(\mathbf{Y}), \ldots, f_{n_{1}}(\mathbf{Y})\right)\right] } \\
& =\mathbb{E}\left[\tilde{\psi}_{1}(\mathbf{X}) \tilde{\psi}_{2}(\mathbf{Y})\right] \leq \mathbb{E}\left[\tilde{\psi}_{1}(\mathbf{X})\right] \mathbb{E}\left[\tilde{\psi}_{2}(\mathbf{Y})\right] \\
& =\mathbb{E}\left[\psi_{1}\left(g_{1}(\mathbf{X}), \ldots, g_{m_{1}}(\mathbf{X})\right)\right] \mathbb{E}\left[\psi_{2}\left(f_{1}(\mathbf{Y}), \ldots, f_{n_{1}}(\mathbf{Y})\right)\right] .
\end{aligned}
$$

Hence $\left(f_{1}(\mathbf{Y}), \ldots, f_{n_{1}}(\mathbf{Y})\right)$ is negatively dependent on $\left(g_{1}(\mathbf{X}), \ldots, g_{m_{1}}(\mathbf{X})\right)$ under $\mathbb{E}$.

The following proposition gives the Chebyshev inequalities in sublinear expectation spaces which have been proved in Chen, Wu and $\mathrm{Li}$ [5]. But the proof there is not valid for the capacities defined by Zhang [21] (see Remark 1.3 in this paper). We give a new proof here which is also valid for the capacities defined by Zhang [21].

Proposition 2.5 (Chebyshev inequalities) Let $X$ be any random variable in $\mathscr{H}$ and $f$ be any nondecreasing positive function in $C_{l, \mathrm{Lip}}(\mathbb{R})$. Then, for any $\in \in \mathbb{R}$,

$$
V(X \geq \epsilon) \leq \frac{\mathbb{E}[f(X)]}{f(\epsilon)}, \quad v(X \geq \epsilon) \leq \frac{\mathcal{E}[f(X)]}{f(\epsilon)} .
$$

Proof Notice that

$$
I_{X \geq \epsilon} \leq \frac{f(X)}{f(\epsilon)} \in \mathscr{H},
$$


then this proposition can be deduced directly from (1) and the positive homogeneity of $\mathbb{E}$ and $\mathcal{E}$.

Theorem 2.6 (Kolmogorov maximal inequality in moment type) Let $1 \leq p \leq 2,\left\{X_{l}\right\}_{l=1}^{n}$ be a sequence of random variables in sublinear expectation space $(\Omega, \mathscr{H}, \mathbb{E})$ with $\mathbb{E}\left[X_{l}\right]=$ $\mathcal{E}\left[X_{l}\right]=0$ for all $l=1, \ldots, n$. If $X_{k}$ is negatively dependent on $\left(X_{k+1}, \ldots, X_{n}\right)$ for all $k=$ $1, \ldots, n-1$, then

$$
\mathbb{E}\left[\max _{1 \leq l \leq n}\left|S_{l}\right|^{p}\right] \leq 2^{3-p} \sum_{l=1}^{n} \mathbb{E}\left[\left|X_{l}\right|^{p}\right] .
$$

Proof By Proposition 2.4, we know that $-X_{k}$ is negatively dependent on $\left(-X_{k+1}, \ldots,-X_{n}\right)$ for all $k=1, \ldots, n-1$. So by Theorem 2.1 in Zhang [21], we have

$$
\mathbb{E}\left[\left|\max _{1 \leq l \leq n} S_{l}\right|^{p}\right] \leq 2^{2-p} \sum_{l=1}^{n} \mathbb{E}\left[\left|X_{l}\right|^{p}\right], \quad \text { and } \quad \mathbb{E}\left[\left|\max _{1 \leq l \leq n}\left(-S_{l}\right)\right|^{p}\right] \leq 2^{2-p} \sum_{l=1}^{n} \mathbb{E}\left[\left|X_{l}\right|^{p}\right]
$$

Notice that

$$
\max _{1 \leq l \leq n}\left|S_{l}\right|^{p}=\left(\max _{1 \leq l \leq n}\left|S_{l}\right|\right)^{p} \leq\left|\max _{1 \leq l \leq n} S_{l}\right|^{p}+\left|\max _{1 \leq l \leq n}\left(-S_{l}\right)\right|^{p},
$$

taking $\mathbb{E}$ in the above inequality, we can obtain (6) from the subadditivity of $\mathbb{E}$.

Theorem 2.7 (Kolmogorov maximal inequality in capacity type) Let $1 \leq p \leq 2,\left\{X_{l}\right\}_{l=1}^{n}$ be a sequence of random variables in sublinear expectation space $(\Omega, \mathscr{H}, \mathbb{E})$ with $\mathbb{E}\left[X_{l}\right]=$ $\mathcal{E}\left[X_{l}\right]=0$ for all $l=1, \ldots, n$. If $X_{k}$ is negatively dependent on $\left(X_{k+1}, \ldots, X_{n}\right)$ for all $k=$ $1, \ldots, n-1$, then, for any $\epsilon>0$,

$$
V\left(\max _{1 \leq l \leq n}\left|S_{l}\right|>\epsilon\right) \leq 2^{3-p} \epsilon^{-p} \sum_{l=1}^{n} \mathbb{E}\left[\left|X_{l}\right|^{p}\right] .
$$

Proof By Proposition 2.5, we have

$$
V\left(\max _{1 \leq l \leq n}\left|S_{l}\right|>\epsilon\right) \leq \epsilon^{-p} \mathbb{E}\left[\max _{1 \leq l \leq n}\left|S_{l}\right|^{p}\right] .
$$

Then inequality (7) can be deduced from Theorem 2.6.

\section{Strong laws of large numbers and the growth rate of partial sums}

In this section and the sequel, we consider the sublinear expectation $\mathbb{E}$ can be represented by

$$
\mathbb{E}[X]=\sup _{P \in \mathcal{P}} E_{P}[X], \quad \text { for all } X \in \mathscr{H},
$$

where $\mathcal{P}$ is a nonempty set of $\sigma$-additive probabilities on $\mathcal{F}$. It is easy to check that

$$
\mathcal{E}[X]=\inf _{P \in \mathcal{P}} E_{P}[X], \quad \text { for all } X \in \mathscr{H},
$$


and $(V, v)$ can be rewritten as

$$
V(A)=\sup _{P \in \mathcal{P}} P(A), \quad v(A)=\inf _{P \in \mathcal{P}} P(A), \quad \text { for all } A \in \mathcal{F} .
$$

Clearly, $V$ is inner continuous and $v$ is outer continuous, that is, $V\left(A_{n}\right) \uparrow V(A)$, if $A_{n} \uparrow A$; and $v\left(A_{n}\right) \downarrow v(A)$, if $A_{n} \downarrow A$, where $A_{n}, A \in \mathcal{F}, n \geq 1$ (see Lemma 2.1 in Chen, Wu and Li [5]). Theorem 3.2 shows that these properties also hold for $\mathbb{E}$ and $\mathcal{E}$.

Definition 3.1 (Definition 3 in Denis, Hu and Peng [7]) A set $B$ is a polar set if $V(B)=0$ and a property holds quasi-surely if it holds outside a polar set.

Theorem 3.2 (The monotone convergence theorem)

(i) If random variables $X_{n} \uparrow X$ and there exists a constant $c$ such that $X_{n} \geq c$ quasi-surely for all $n \geq 1$, then $\mathbb{E}\left[X_{n}\right] \uparrow \mathbb{E}[X]$.

(ii) If random variables $Y_{n} \downarrow Y$ and there exists a constant $c$ such that $Y_{n} \leq c$ quasi-surely for all $n \geq 1$, then $\mathcal{E}\left[Y_{n}\right] \downarrow \mathcal{E}[Y]$.

Proof It is easy to see that (i) is equivalent to (ii). So we only prove (i). By the monotonicity of $\mathbb{E}$, we know $\mathbb{E}\left[X_{n}\right]$ nondecreasing and $\mathbb{E}[X] \geq \lim _{n \rightarrow \infty} \mathbb{E}\left[X_{n}\right]$. On the other hand, it follows from the classical monotone convergence theorem that

$$
\mathbb{E}[X]=\sup _{P \in \mathcal{P}} E_{P}[X]=\sup _{P \in \mathcal{P}} \lim _{n \rightarrow \infty} E_{P}\left[X_{n}\right] \leq \lim _{n \rightarrow \infty} \mathbb{E}\left[X_{n}\right] .
$$

Therefore (i) is proved.

Let $\varphi$ be a positive function satisfying

$$
\sum_{n=1}^{\infty} \frac{\varphi(n)}{n^{2}}<\infty \quad \text { and } \quad 0<\varphi(x) \uparrow \infty \quad \text { on }[c, \infty) \text { for some } c>0
$$

For convenience, we define $\frac{1}{0}=\infty, \frac{1}{\infty}=0$ and $\varphi(\infty)=\infty$.

Remark 3.3 For any $0<\delta<1$ and $\alpha \in \mathbb{R}$, functions $|x|^{\delta}$ and $|x|^{\delta}(\log |x|)^{\alpha}$ satisfy (8).

Lemma 3.4 (Lemma 1 in Sung, Hu and Volodin [17]) Let $\left\{a_{n}\right\}_{n \geq 1}$ be a sequence of nonnegative real numbers such that $a_{n}>0$ for infinitely many $n$. Let $v_{n}=\sum_{i=n}^{\infty} a_{i}$ for $n \in \mathbb{N}^{*}$ and $\varphi$ be a positive function satisfying (8). If $\sum_{n=1}^{\infty} a_{n}<\infty$, then $\sum_{n=1}^{\infty} a_{n} \varphi\left(\frac{1}{v_{n}}\right)<\infty$.

Proposition 3.5 Let $\left\{b_{n}\right\}_{n \geq 1}$ be a nondecreasing unbounded sequence of positive numbers, $\left\{\alpha_{n}\right\}_{n \geq 1}$ be a sequence of non-negative real numbers, and $\varphi$ be a positive function satisfying (8). Let $r$ be any fixed positive number and for $n \in \mathbb{N}^{*}$,

$$
v_{n}=\sum_{i=n}^{\infty} \alpha_{i} b_{i}^{-r} \quad \text { and } \quad \beta_{n}=\max _{1 \leq i \leq n} b_{i} \varphi\left(\frac{1}{v_{i}}\right)^{-1 / r} .
$$

If $\sum_{n=1}^{\infty} \alpha_{n} b_{n}^{-r}<\infty$, then $\sum_{n=1}^{\infty} \alpha_{n} \beta_{n}^{-r}<\infty$ and $\lim _{n \rightarrow \infty} \frac{\beta_{n}}{b_{n}}=0$. 
Proof Firstly, we consider that there exists an integer $m$ such that $\alpha_{n}=0$ for all $n \geq m$, thus $\beta_{n}=\max _{1 \leq i \leq m} b_{i} \varphi\left(\frac{1}{v_{i}}\right)^{-1 / r}$ for $n \geq m$. Obviously, $\sum_{n=1}^{\infty} \alpha_{n} \beta_{n}^{-r}<\infty$ and we can get $\lim _{n \rightarrow \infty} \frac{\beta_{n}}{b_{n}}=0$ from $\lim _{n \rightarrow \infty} b_{n}=\infty$.

Secondly, we consider that $\alpha_{n}>0$ for infinitely many $n$. It is easy to see that $\beta_{n} \leq \beta_{n+1}$ and $\beta_{n} \geq b_{n} \varphi\left(\frac{1}{v_{n}}\right)^{-1 / r}$, for $n \in \mathbb{N}^{*}$. Then, by Lemma 3.4, we have

$$
\sum_{n=1}^{\infty} \alpha_{n} \beta_{n}^{-r} \leq \sum_{n=1}^{\infty} \alpha_{n} b_{n}^{-r} \varphi\left(\frac{1}{v_{n}}\right)<\infty
$$

Therefore, for any $k \leq n$, we have

$$
\begin{aligned}
\frac{\beta_{n}}{b_{n}} & \leq \frac{\max _{1 \leq i \leq k} b_{i} \varphi\left(\frac{1}{v_{i}}\right)^{-1 / r}}{b_{n}}+\frac{\max _{k \leq i \leq n} b_{i} \varphi\left(\frac{1}{v_{i}}\right)^{-1 / r}}{b_{n}} \\
& \leq \frac{\max _{1 \leq i \leq k} b_{i} \varphi\left(\frac{1}{v_{i}}\right)^{-1 / r}}{b_{n}}+\varphi\left(\frac{1}{v_{k}}\right)^{-1 / r} .
\end{aligned}
$$

Let $n \rightarrow \infty$, we get $\limsup _{n \rightarrow \infty} \frac{\beta_{n}}{b_{n}} \leq \varphi\left(\frac{1}{v_{k}}\right)^{-1 / r}$ since $\lim _{n \rightarrow \infty} b_{n}=\infty$. Then let $k \rightarrow \infty$, we have $\lim \sup _{n \rightarrow \infty} \frac{\beta_{n}}{b_{n}} \leq 0$ since $\lim _{n \rightarrow \infty} v_{n}=0$ and $\varphi(x) \uparrow \infty$ as $x \uparrow \infty$. Hence $\lim _{n \rightarrow \infty} \frac{\beta_{n}}{b_{n}}=0$. The proof of this proposition is completed.

Theorems 3.6, 3.8 and 3.9 give several strong laws of large numbers and the growth rate of the partial sums for general random variables in sublinear expectation spaces.

Theorem 3.6 Let $\left\{b_{n}\right\}_{n \geq 1}$ be a nondecreasing unbounded sequence of positive numbers and $\left\{\alpha_{n}\right\}_{n \geq 1}$ be a sequence of non-negative numbers. Let $r$ and $c$ be any fixed positive numbers and $\varphi$ be any positive function satisfying (8). For $n \in \mathbb{N}^{*}$, let

$$
v_{n}=\sum_{i=n}^{\infty} \alpha_{i} b_{i}^{-r} \quad \text { and } \quad \beta_{n}=\max _{1 \leq i \leq n} b_{i} \varphi\left(\frac{1}{v_{i}}\right)^{-1 / r} .
$$

If for each $n \in \mathbb{N}^{*}$,

$$
\mathbb{E}\left[\max _{1 \leq i \leq n}\left|S_{i}\right|^{r}\right] \leq c \sum_{i=1}^{n} \alpha_{i}
$$

and $\sum_{n=1}^{\infty} \alpha_{n} b_{n}^{-r}<\infty$, then $\lim _{n \rightarrow \infty} \frac{S_{n}}{b_{n}}=0$ quasi-surely with the convergence rate $\frac{S_{n}}{b_{n}}=O\left(\frac{\beta_{n}}{b_{n}}\right)$ quasi-surely.

Proof Step 1. We firstly consider that there exists an integer $m$ such that $\alpha_{n}=0$ for all $n \geq m$, then $\beta_{n}=\max _{1 \leq i \leq m} b_{i} \varphi\left(\frac{1}{v_{i}}\right)^{-1 / r}$ for $n \geq m$.

In this case, the sequence $\left\{\beta_{n}\right\}_{n \geq 1}$ is bounded and it follows from the monotone convergence theorem (Theorem 3.2) that

$$
\mathbb{E}\left[\sup _{n \geq 1}\left|S_{n}\right|^{r}\right]=\lim _{n \rightarrow \infty} \mathbb{E}\left[\max _{1 \leq i \leq n}\left|S_{i}\right|^{r}\right] \leq c \sum_{i=1}^{\infty} \alpha_{i}<\infty
$$

thus $\sup _{n \geq 1}\left|S_{n}\right|<\infty$ quasi-surely. 
Therefore $\lim _{n \rightarrow \infty} \frac{S_{n}}{b_{n}}=0$ quasi-surely since $\lim _{n \rightarrow \infty} b_{n}=\infty$. Because of $\frac{S_{n}}{b_{n}}=\frac{S_{n}}{\beta_{n}} \frac{\beta_{n}}{b_{n}}$, we obtain the convergence rate of $\frac{S_{n}}{b_{n}}=O\left(\frac{\beta_{n}}{b_{n}}\right)$ quasi-surely.

Step 2. We consider that $\alpha_{n}>0$ for infinitely many $n$. By Proposition 3.5, we have $\sum_{n=1}^{\infty} \alpha_{n} \beta_{n}^{-r}<\infty$, and $\lim _{n \rightarrow \infty} \beta_{n} b_{n}^{-1}=0$. It follows from Theorem 2.1 that

$$
\mathbb{E}\left[\max _{1 \leq l \leq n}\left|\frac{S_{l}}{\beta_{l}}\right|^{r}\right] \leq 4 c \sum_{l=1}^{n} \frac{\alpha_{l}}{\beta_{l}^{r}} \leq 4 c \sum_{l=1}^{\infty} \frac{\alpha_{l}}{\beta_{l}^{r}}<\infty .
$$

By the monotone convergence theorem (Theorem 3.2), we have

$$
\mathbb{E}\left[\sup _{n \geq 1}\left|\frac{S_{n}}{\beta_{n}}\right|^{r}\right]=\lim _{n \rightarrow \infty} \mathbb{E}\left[\max _{1 \leq l \leq n}\left|\frac{S_{l}}{\beta_{l}}\right|^{r}\right] \leq 4 c \sum_{l=1}^{\infty} \frac{\alpha_{l}}{\beta_{l}^{r}}<\infty
$$

So $\sup _{n \geq 1}\left|\frac{S_{n}}{\beta_{n}}\right|<\infty$ quasi-surely and

$$
0 \leq\left|\frac{S_{n}}{b_{n}}\right| \leq\left|\frac{\beta_{n}}{b_{n}}\right| \sup _{n \geq 1}\left|\frac{S_{n}}{\beta_{n}}\right| \text {. }
$$

Hence, $\lim _{n \rightarrow \infty} \frac{S_{n}}{b_{n}}=0$ quasi-surely and $\frac{S_{n}}{b_{n}}=O\left(\frac{\beta_{n}}{b_{n}}\right)$ quasi-surely. The proof of this theorem is completed.

Proposition 3.7 Under the assumptions of Theorem 3.6, the following statements hold:

(i) if the sequence $\left\{\beta_{n}\right\}_{n \geq 1}$ is bounded, then $\frac{S_{n}}{\beta_{n}}=O(1)$ quasi-surely;

(ii) if the sequence $\left\{\beta_{n}\right\}_{n \geq 1}$ is unbounded, then $\frac{S_{n}}{\beta_{n}}=o(1)$ quasi-surely.

Proof (i) Assume that $\left\{\beta_{n}\right\}_{n \geq 1}$ is bounded by a constant $D>0$. Then

$$
\sum_{n=1}^{\infty} \alpha_{n} \leq D^{r} \sum_{n=1}^{\infty} \alpha_{n} \beta_{n}^{-r}<\infty .
$$

It follows from the monotone convergence theorem (Theorem 3.2) that

$$
\mathbb{E}\left[\sup _{n \geq 1}\left|S_{n}\right|^{r}\right]=\lim _{n \rightarrow \infty} \mathbb{E}\left[\max _{1 \leq l \leq n}\left|S_{l}\right|^{r}\right] \leq c \sum_{l=1}^{\infty} \alpha_{l}<\infty
$$

Therefore $\sup _{n \geq 1}\left|S_{n}\right|<\infty$ quasi-surely. Since $\left\{\beta_{n}\right\}_{n \geq 1}$ is bounded, we obtain $\frac{S_{n}}{\beta_{n}}=O(1)$ quasi-surely.

(ii) We turn to the case that $\left\{\beta_{n}\right\}_{n \geq 1}$ is unbounded. Now $\left\{\beta_{n}\right\}_{n \geq 1}$ is a nondecreasing unbounded sequence of positive numbers. It follows from Proposition 3.5 that $\sum_{n=1}^{\infty} \alpha_{n} \beta_{n}^{-r}<$ $\infty$. Then, by Theorem 3.6, we get $\lim _{n \rightarrow \infty} \frac{S_{n}}{\beta_{n}}=0$ quasi-surely, that is, $\frac{S_{n}}{\beta_{n}}=o(1)$ quasisurely.

Theorem 3.8 Under the assumptions of Theorem 3.6, the following statements hold:

(i) if the sequence $\left\{\beta_{n}\right\}_{n \geq 1}$ is bounded, then $\frac{S_{n}}{b_{n}}=O\left(\frac{\beta_{n}}{b_{n}}\right)$ quasi-surely;

(ii) if the sequence $\left\{\beta_{n}\right\}_{n \geq 1}$ is unbounded, then $\frac{S_{n}}{b_{n}}=o\left(\frac{\beta_{n}}{b_{n}}\right)$ quasi-surely.

Proof Due to $\frac{S_{n}}{b_{n}}=\frac{S_{n}}{\beta_{n}} \frac{\beta_{n}}{b_{n}}$, these two statements can easily be got from Theorem 3.6 and Proposition 3.7. 
Theorem 3.9 Let $\left\{b_{n}\right\}_{n \geq 1}$ be a nondecreasing unbounded sequence of positive numbers and $\left\{\alpha_{n}\right\}_{n \geq 1}$ be a sequence of non-negative numbers and $r$ be any fixed positive number. For $n \in \mathbb{N}$, let

$$
v_{n}=\sum_{i=n}^{\infty} \alpha_{i} b_{i}^{-r} \text { and } \beta_{n}=\max _{1 \leq i \leq n} b_{i} \varphi\left(\frac{1}{v_{i}}\right)^{-1 / r} .
$$

Assume that $\sum_{n=1}^{\infty} \alpha_{n} b_{n}^{-r}<\infty$ and there exists a constant $c>0$ such that for any $n \in \mathbb{N}^{*}$ and any $\epsilon>0$

$$
V\left(\max _{1 \leq i \leq n}\left|S_{i}\right| \geq \epsilon\right) \leq c \epsilon^{-r} \sum_{i=1}^{n} \alpha_{i}
$$

Then the following statements hold:

(i) $\lim _{n \rightarrow \infty} \frac{S_{n}}{b_{n}}=0$ quasi-surely and $\frac{S_{n}}{b_{n}}=O\left(\frac{\beta_{n}}{b_{n}}\right)$ quasi-surely;

(ii) if the sequence $\left\{\beta_{n}\right\}_{n \geq 1}$ is bounded, then $\frac{S_{n}}{\beta_{n}}=O(1)$ quasi-surely and $\frac{S_{n}}{b_{n}}=O\left(\frac{\beta_{n}}{b_{n}}\right)$ quasi-surely;

(iii) if the sequence $\left\{\beta_{n}\right\}_{n \geq 1}$ is unbounded, then $\frac{S_{n}}{\beta_{n}}=o(1)$ quasi-surely and $\frac{S_{n}}{b_{n}}=o\left(\frac{\beta_{n}}{b_{n}}\right)$ quasi-surely.

Proof (i) By Theorem 2.2, we have for any $n \in \mathbb{N}^{*}$ and any $\epsilon>0$

$$
V\left(\max _{1 \leq i \leq n}\left|\frac{S_{i}}{\beta_{i}}\right| \geq \epsilon\right) \leq 4 c \epsilon^{-r} \sum_{i=1}^{n} \frac{\alpha_{i}}{\beta_{i}^{r}} .
$$

So for any $k \in \mathbb{N}^{*}$, it follows from the inner continuity of $V$ that

$$
V\left(\sup _{n \geq 1}\left|\frac{S_{n}}{\beta_{n}}\right|>k\right) \leq \lim _{n \rightarrow \infty} V\left(\max _{1 \leq i \leq n}\left|\frac{S_{i}}{\beta_{i}}\right| \geq k\right) \leq 4 c k^{-r} \sum_{i=1}^{\infty} \frac{\alpha_{i}}{\beta_{i}^{r}} .
$$

From Proposition 3.5, we have $\sum_{i=1}^{\infty} \alpha_{i} \beta_{i}^{-r}<\infty$. Therefore

$$
\lim _{k \rightarrow \infty} V\left(\sup _{n \geq 1}\left|\frac{S_{n}}{\beta_{n}}\right|>k\right)=0
$$

By the monotonicity of $V$, we have

$$
V\left(\sup _{n \geq 1}\left|\frac{S_{n}}{\beta_{n}}\right|=\infty\right)=V\left(\bigcap_{k=1}^{\infty}\left\{\sup _{n \geq 1}\left|\frac{S_{n}}{\beta_{n}}\right|>k\right\}\right) \leq \lim _{k \rightarrow \infty} V\left(\sup _{n \geq 1}\left|\frac{S_{n}}{\beta_{n}}\right|>k\right)=0 .
$$

Consequently $\sup _{n \geq 1}\left|S_{n}\right| / \beta_{n}<\infty$ quasi-surely. Due to

$$
\frac{\left|S_{n}\right|}{b_{n}}=\frac{\left|S_{n}\right|}{\beta_{n}} \frac{\beta_{n}}{b_{n}}
$$

and Proposition 3.5, we have $\lim _{n \rightarrow \infty} \frac{S_{n}}{b_{n}}=0$ and $\frac{S_{n}}{b_{n}}=O\left(\frac{\beta_{n}}{b_{n}}\right)$ quasi-surely. 
(ii) If the sequence $\left\{\beta_{n}\right\}_{n \geq 1}$ is bounded by constant $D>0$, then

$$
\sum_{n=1}^{\infty} \alpha_{n} \leq D^{r} \sum_{n=1}^{\infty} \alpha_{n} \beta_{n}^{-r}<\infty
$$

So for any $k \in \mathbb{N}^{*}$, it follows from the inner continuity of $V$ and (10) that

$$
V\left(\sup _{n \geq 1}\left|S_{n}\right|>k\right) \leq \lim _{n \rightarrow \infty} V\left(\max _{1 \leq i \leq n}\left|S_{i}\right| \geq k\right) \leq c k^{-r} \sum_{i=1}^{\infty} \alpha_{i}
$$

Therefore

$$
\lim _{k \rightarrow \infty} V\left(\sup _{n \geq 1}\left|S_{n}\right|>k\right)=0
$$

By the monotonicity of $V$, we have

$$
V\left(\sup _{n \geq 1}\left|S_{n}\right|=\infty\right)=V\left(\bigcap_{k=1}^{\infty}\left\{\sup _{n \geq 1}\left|S_{n}\right|>k\right\}\right) \leq \lim _{k \rightarrow \infty} V\left(\sup _{n \geq 1}\left|S_{n}\right|>k\right)=0 .
$$

Consequently $\sup _{n \geq 1}\left|S_{n}\right|<\infty$ quasi-surely. Hence, $\frac{S_{n}}{\beta_{n}}=O(1)$ quasi-surely and $\frac{S_{n}}{b_{n}}=O\left(\frac{\beta_{n}}{b_{n}}\right)$ quasi-surely.

(iii) If the sequence $\left\{\beta_{n}\right\}_{n \geq 1}$ is unbounded, then, by Proposition 3.5 we have

$$
\sum_{n=1}^{\infty} \alpha_{n} \beta_{n}^{-r}<\infty
$$

From (i) of this theorem, we get $\lim _{n \rightarrow \infty} S_{n} / \beta_{n}=0$ quasi-surely, and thus $S_{n} / b_{n}=o\left(\beta_{n} / b_{n}\right)$ quasi-surely.

The result of Theorem 3.8 is more precise than Theorem 3.6. When the sublinear expectation space degenerates to the classical probability space, Theorem 3.8 and Theorem 3.9 give more precise results than Theorem 2.1 in Fazekas and Klesov [9], Lemma $1.2 \mathrm{in} \mathrm{Hu}$ and Hu [10], Theorem 3.4 in Tómács [19] and Theorem 2.4 in Tómács and Líbor [20].

Theorem 3.10 (Strong law of large numbers for negatively dependent random variables) Let $1 \leq p \leq 2,\left\{X_{n}\right\}_{n \geq 1}$ be a sequence of random variables in sublinear expectation space $(\Omega, \mathscr{H}, \mathbb{E})$ with $\mathbb{E}\left[X_{n}\right]=\mathcal{E}\left[X_{n}\right]=0$ for all $n \in \mathbb{N}^{*}$. If $X_{k}$ is negatively dependent on $\left(X_{k+1}, \ldots, X_{k+n}\right)$ for all $n, k \in \mathbb{N}^{*}$ and $\left\{b_{n}\right\}_{n \geq 1}$ is a nondecreasing unbounded sequence of positive numbers with

$$
\sum_{n=1}^{\infty} \frac{\mathbb{E}\left[\left|X_{n}\right|^{p}\right]}{b_{n}^{p}}<\infty
$$

then

$$
\lim _{n \rightarrow \infty} \frac{S_{n}}{b_{n}}=0 \quad \text { quasi-surely }
$$


with the convergence rate $\frac{S_{n}}{b_{n}}=O\left(\frac{\beta_{n}}{b_{n}}\right)$ when $\left\{\beta_{n}\right\}_{n \geq 1}$ is bounded, $\frac{S_{n}}{b_{n}}=o\left(\frac{\beta_{n}}{b_{n}}\right)$ when $\left\{\beta_{n}\right\}_{n \geq 1}$ is unbounded, where for $n \in \mathbb{N}^{*}$,

$$
\beta_{n}=\max _{1 \leq i \leq n} b_{i} \varphi\left(\frac{1}{v_{i}}\right)^{-1 / p}, \quad v_{n}=\sum_{i=n}^{\infty} \mathbb{E}\left[\left|X_{i}\right|^{p}\right] b_{i}^{-p}
$$

and $\varphi$ is any positive function satisfying (8).

Proof Set $\alpha_{k}=\mathbb{E}\left[\left|X_{k}\right|^{p}\right]$ for all $k \in \mathbb{N}^{*}$, then, by Theorem 2.6, we have

$$
\mathbb{E}\left[\max _{1 \leq k \leq n}\left|S_{k}\right|^{p}\right] \leq 2^{3-p} \sum_{k=1}^{n} \alpha_{k}, \quad n \geq 1 .
$$

Due to

$$
\sum_{n=1}^{\infty} \frac{\alpha_{n}}{b_{n}^{p}}=\sum_{n=1}^{\infty} \frac{\mathbb{E}\left[\left|X_{n}\right|^{p}\right]}{b_{n}^{p}}<\infty,
$$

we can deduce this theorem from Theorem 3.6 and 3.8 .

\section{An application to the logarithmically weighted sums}

By using Theorem 3.6 and 3.8 to the logarithmically weighted sums, we can get Theorem 4.2, which sharpens the result of Theorem 8.1 in Fazekas and Klesov [9] and Theorem $2.5 \mathrm{in} \mathrm{Hu}$ and $\mathrm{Hu}[10]$ under the same condition in the classical probability theory and extends it to the sublinear expectation space. Some of our idea for obtaining Theorem 4.2 come from these papers.

Lemma 4.1 (Lemma 8.1 in Fazekas and Klesov [9]) Set $g(i, j)=\sum_{k=i}^{j} \frac{1}{k}$ for $i \leq j$. Then, for any $0<\beta<1$ and $1<\gamma<2$, we have

$$
\sum_{k=i}^{j} \sum_{l=i}^{k} \frac{1}{k^{1+\beta}} \frac{1}{l^{1-\beta}} \leq \frac{2}{\beta} g^{\gamma}(i, j) .
$$

Theorem 4.2 Let $\left\{X_{n}\right\}_{n \geq 1}$ satisfy the following condition (11):

there exist constants $\beta>0, c>0$ such that

$$
\left|\mathbb{E}\left[\left(X_{k}-\mathbb{E}\left[X_{k}\right]\right)\left(X_{l}-\mathbb{E}\left[X_{l}\right]\right)\right]\right| \leq c\left(\frac{l}{k}\right)^{\beta}, \quad 1 \leq l \leq k .
$$

Then

$$
\lim _{n \rightarrow \infty} \frac{1}{\log n} \sum_{k=1}^{n} \frac{X_{k}-\mathbb{E}\left[X_{k}\right]}{k}=0 \quad \text { quasi-surely }
$$

and for any $\epsilon \in(0,1 / 2)$,

$$
\frac{1}{\log n} \sum_{k=1}^{n} \frac{X_{k}-\mathbb{E}\left[X_{k}\right]}{k}=o\left(\frac{1}{(\log n)^{\epsilon}}\right) \text { quasi-surely. }
$$


Proof Without loss of generality we may assume $0<\beta<1$. Using the assumption (11) and Lemma 4.1 we have for all $i<j, 1<\gamma<2$

$$
\mathbb{E}\left[\left(\sum_{k=i}^{j} \frac{X_{k}-\mathbb{E}\left[X_{k}\right]}{k}\right)^{2}\right] \leq 2 c \sum_{k=i}^{j} \sum_{l=i}^{k} \frac{1}{k^{1+\beta}} \frac{1}{l^{1-\beta}} \leq \frac{4 c}{\beta}\left(\sum_{k=i}^{j} \frac{1}{k}\right)^{\gamma} .
$$

By Theorem 1 in Longnecker and Serfling [11], we get, for every $P \in \mathcal{P}$,

$$
E_{P}\left[\max _{1 \leq i \leq n}\left|\sum_{k=1}^{i} \frac{X_{k}-\mathbb{E}\left[X_{k}\right]}{k}\right|^{2}\right] \leq A_{2, \gamma} \frac{4 c}{\beta}\left(\sum_{k=1}^{n} \frac{1}{k}\right)^{\gamma},
$$

then

$$
\mathbb{E}\left[\max _{1 \leq i \leq n}\left|\sum_{k=1}^{i} \frac{X_{k}-\mathbb{E}\left[X_{k}\right]}{k}\right|^{2}\right] \leq A_{2, \gamma} \frac{4 c}{\beta}\left(\sum_{k=1}^{n} \frac{1}{k}\right)^{\gamma},
$$

where $A_{2, \gamma}$ is a constant defined in Theorem 1 of Longnecker and Serfling [11]. Since $\sum_{k=1}^{n} 1 / k=O(1) \log n$ as $n \rightarrow \infty$, we denote

$$
\alpha_{n}=(\log (n+1))^{\gamma}-(\log n)^{\gamma}
$$

and then by (13) we have for all $n \geq 1$

$$
\mathbb{E}\left[\max _{1 \leq i \leq n}\left|\sum_{k=1}^{i} \frac{X_{k}-\mathbb{E}\left[X_{k}\right]}{k}\right|^{2}\right] \leq c_{1} \sum_{k=1}^{n} \alpha_{k}
$$

where $c_{1}$ is a positive constant. Due to $\lim _{n \rightarrow \infty} \gamma n^{-1}(\log n)^{\gamma-1} / \alpha_{n}=1$, there exists $n_{0} \geq 3$, $c_{2}>0$ and $c_{3}>0$ such that, for all $n \geq n_{0}$,

$$
c_{2} n^{-1}(\log n)^{\gamma-1} \leq \alpha_{n} \leq c_{3} n^{-1}(\log n)^{\gamma-1} .
$$

Take $b_{n}=\log (n \vee 2)$, then

$$
v_{1}=\sum_{n=1}^{\infty} \alpha_{n} b_{n}^{-2}<\infty
$$

Hence by Theorem 3.6, (12) holds.

Meanwhile, for $n \geq n_{0}$, we have

$$
\frac{c_{2}}{2-\gamma}(\log n)^{\gamma-2} \leq v_{n}=\sum_{i=n}^{\infty} \alpha_{i} b_{i}^{-2} \leq \frac{c_{3}}{2-\gamma}(\log (n-1))^{\gamma-2} .
$$

Therefore, for any $\delta \in(0,1), n \geq n_{0}$,

$$
\beta_{n}=\max _{1 \leq i \leq n} b_{i} v_{i}^{\delta / 2} \geq\left(\frac{c_{2}}{2-\gamma}\right)^{\delta / 2}(\log n)^{1-(2-\gamma) \delta / 2}
$$


which implies $\left\{\beta_{n}\right\}_{n \geq 1}$ is unbounded since $1-(2-\gamma) \delta / 2 \in(0,1 / 2)$. Therefore, by Theorem 3.8, the convergence rate of $\frac{1}{\log n} \sum_{k=1}^{n} \frac{X_{k}-\mathbb{E}\left[X_{k}\right]}{k}$ is $o\left(\beta_{n} / b_{n}\right)$ quasi-surely.

On the other hand, for $n \geq n_{0}$, we have

$$
\begin{aligned}
\beta_{n} & =\max _{1 \leq i \leq n} b_{i} v_{i}^{\delta / 2}=\max \left\{\beta_{n_{0}}, \max _{n_{0} \leq i \leq n} b_{i} \delta_{i}^{\delta / 2}\right\} \\
& \leq \max \left\{\beta_{n_{0}},\left(\frac{c_{3}}{2-\gamma}\right)^{\delta / 2}(\log n)(\log (n-1))^{(\gamma-2) \delta / 2}\right\} \\
& \leq \max \left\{\beta_{n_{0}},\left(\frac{c_{3}}{2-\gamma}\right)^{\delta / 2}\left(\frac{\log 3}{\log 2}\right)^{(2-\gamma) \delta / 2}(\log n)^{1-(2-\gamma) \delta / 2}\right\} .
\end{aligned}
$$

Since $\left\{\beta_{n}\right\}_{n \geq 1}$ is unbounded, for sufficient large $n$, we have

$$
\frac{\beta_{n}}{b_{n}} \leq c_{4}(\log n)^{-(2-\gamma) \delta / 2}
$$

where $c_{4}=\left(\frac{c_{3}}{2-\gamma}\right)^{\delta / 2}\left(\frac{\log 3}{\log 2}\right)^{(2-\gamma) \delta / 2}$. Let $\epsilon=(2-\gamma) \delta / 2$, then $\epsilon$ can be any value in $(0,1 / 2)$ since $\delta$ is an arbitrary number in $(0,1)$ and $\frac{1}{\log n} \sum_{k=1}^{n} \frac{X_{k}-\mathbb{E}\left[X_{k}\right]}{k}=o\left(\frac{1}{(\log n)^{\epsilon}}\right)$ quasi-surely.

Throughout the sequel of this section, the sublinear expectation spaces and the random variable sequence $\left\{X_{n}\right\}_{n \geq 1}$ are further supposed to satisfy the following two assumptions.

Assumption 1 The sublinear expectation spaces $(\Omega, \mathscr{H}, \mathbb{E})$ and $(\widetilde{\Omega}, \widetilde{\mathscr{H}}, \widetilde{\mathbb{E}})$ satisfy for all $X \in \mathscr{H}\left(\right.$ or $\widetilde{\mathscr{H}}$ ) and $f_{n} \in C_{b, \text { Lip }}(\mathbb{R}), f_{n} \downarrow 0: \mathbb{E}($ or $\widetilde{\mathbb{E}})\left[f_{n}(X)\right] \downarrow 0$.

Assumption 2 Having fixed the ratio $\lambda \geq 1$ as a constant, the sequence $\left\{X_{n}\right\}_{n \geq 1}$ is a sequence of independent random variables in any fixed sublinear expectation space $(\Omega, \mathscr{H}, \mathbb{E})$ with $\mathbb{E}\left[X_{n}\right]=\mathcal{E}\left[X_{n}\right]=0, \bar{\sigma}_{n}=\sqrt{\mathbb{E}\left[X_{n}^{2}\right]}$ and $\underline{\sigma}_{n}=\sqrt{\mathcal{E}\left[X_{n}^{2}\right]}$, for $n \geq 1$, and $0<$ $\inf _{n \geq 1} \underline{\sigma}_{n}^{2} \leq \sup _{n \geq 1} \bar{\sigma}_{n}^{2}<\infty$. For $n \geq 1$, denote $S_{0}=0, S_{n}=\sum_{i=1}^{n} X_{i}$ and $\sigma_{n}:=\frac{\underline{\sigma}_{n}+\bar{\sigma}_{n}}{2}, \lambda_{n}:=$ $\frac{\bar{\sigma}_{n}}{\underline{\sigma}_{n}} \equiv \lambda, B_{n}:=\sqrt{\sum_{i=1}^{n} \sigma_{i}^{2}}, W_{n}:=\frac{S_{n}}{B_{n}}$.

A random variable $\xi$ is G-normal distributed (denoted by $\xi \sim N\left(0,\left[\sigma^{2}, \bar{\sigma}^{2}\right]\right)$ ) under a sublinear expectation $\widetilde{\mathbb{E}}$, if and only if for any $f \in C_{b, \text { Lip }}(\mathbb{R})$, the function $u(t, x)=\widetilde{\mathbb{E}}[f(x+$ $\sqrt{t} \xi)](x \in \mathbb{R}, t \geq 0)$ is the unique viscosity solution of the following $G$-heat equation:

$$
\left\{\begin{array}{l}
\partial_{t} u-G\left(\partial_{x x}^{2} u\right)=0, \quad(x, t) \in \mathbb{R} \times(0, \infty), \\
u(0, x)=f(x)
\end{array}\right.
$$

where $G(a)=\frac{1}{2} \widetilde{\mathbb{E}}\left[a \xi^{2}\right], a \in \mathbb{R}$, is determined by the variances $\bar{\sigma}^{2}:=\widetilde{\mathbb{E}}\left[\xi^{2}\right]$ and $\underline{\sigma}^{2}:=\widetilde{\mathcal{E}}\left[\xi^{2}\right]$. If $\bar{\sigma}^{2}=\underline{\sigma}^{2}$, then $G$-normal distribution is just the classical normal distribution $N\left(0, \bar{\sigma}^{2}\right)$.

Lemma 4.3 (Theorem 5.1 in Song [16]) Let $(\Omega, \mathscr{H}, \mathbb{E})$ and $(\widetilde{\Omega}, \widetilde{\mathscr{H}}, \widetilde{\mathbb{E}})$ be sublinear expectation spaces satisfying Assumption 1 and $\left\{X_{n}\right\}_{n \geq 1}$ be a sequence of random variables in $(\Omega, \mathscr{H}, \mathbb{E})$ satisfying Assumption 2 . Then there exist a constant $\alpha \in(0,1)$, depending on $\lambda$, and a constant $C_{\alpha, \lambda}>0$, depending on $\alpha, \lambda$ such that, for any $n \geq 1$,

$$
\sup _{|f|_{\text {Lip }} \leq 1}\left|\mathbb{E}\left[f\left(W_{n}\right)\right]-\widetilde{\mathbb{E}}[f(\xi)]\right| \leq C_{\alpha, \lambda} \sup _{1 \leq i \leq n}\left\{\frac{\mathbb{E}\left[\left|X_{i}\right|^{2+\alpha}\right]}{\sigma_{i}^{2+\alpha}}\left(\frac{\sigma_{i}}{B_{n}}\right)^{\alpha}\right\}
$$


where $\xi$ is G-normal distribution under $\widetilde{\mathbb{E}}$ with the fixed $\lambda$ and $\sqrt{\mathbb{E}\left[\xi^{2}\right]}=\frac{2 \lambda}{1+\lambda}, \sqrt{\mathcal{E}\left[\xi^{2}\right]}=\frac{2}{1+\lambda}$ and $|f|_{\text {Lip }}$ is the Lipschitz constant off .

Theorem 4.4 Let $(\Omega, \mathscr{H}, \mathbb{E})$ and $(\widetilde{\Omega}, \widetilde{\mathscr{H}}, \widetilde{\mathbb{E}})$ be sublinear expectation spaces satisfying Assumption 1 and $\left\{X_{n}\right\}_{n \geq 1}$ be a sequence of random variables in $(\Omega, \mathscr{H}, \mathbb{E})$ satisfying Assumption 2. For the $\alpha$ in Lemma 4.3, if $\sup _{i \geq 1} \mathbb{E}\left[\left|X_{i}\right|^{2+\alpha}\right]<\infty$, then, for any $f \in C_{b, \text { Lip }}(\mathbb{R})$, we have

$$
\lim _{n \rightarrow \infty} \frac{1}{\log n} \sum_{k=1}^{n} \frac{\mathbb{E}\left[f\left(W_{k}\right)\right]}{k}=\widetilde{\mathbb{E}}[f(\xi)]
$$

and

$$
\lim _{n \rightarrow \infty} \frac{1}{\log n} \sum_{k=1}^{n} \frac{\mathcal{E}\left[f\left(W_{k}\right)\right]}{k}=\widetilde{\mathcal{E}}[f(\xi)],
$$

and the convergence rate is $O\left(\frac{1}{\log n}\right)$, where $\xi$ is G-normal distribution under $\widetilde{\mathbb{E}}$ with the fixed $\lambda$ and $\sqrt{\widetilde{\mathbb{E}}\left[\xi^{2}\right]}=\frac{2 \lambda}{1+\lambda}, \sqrt{\widetilde{\mathcal{E}}\left[\xi^{2}\right]}=\frac{2}{1+\lambda}$.

Proof We can get (15) by considering $-f$ in (14). So we only need to prove (14). It follows from Lemma 4.3 that

$$
\begin{aligned}
& \limsup _{n \rightarrow \infty}\left|\frac{1}{\log n} \sum_{k=1}^{n} \frac{\mathbb{E}\left[f\left(W_{k}\right)\right]}{k}-\widetilde{\mathbb{E}}[f(\xi)]\right| \\
& \quad=\limsup _{n \rightarrow \infty}\left|\frac{1}{\log n} \sum_{k=1}^{n} \frac{\mathbb{E}\left[f\left(W_{k}\right)\right]-\widetilde{\mathbb{E}}[f(\xi)]}{k}\right| \\
& \quad \leq \limsup _{n \rightarrow \infty} \frac{1}{\log n} \sum_{k=1}^{n} \frac{\left|\mathbb{E}\left[f\left(W_{k}\right)\right]-\widetilde{\mathbb{E}}[f(\xi)]\right|}{k} \\
& \leq \limsup _{n \rightarrow \infty} \frac{1}{\log n} \sum_{k=1}^{n} \frac{|f|_{\text {Lip }} C_{\alpha, \lambda}}{k} \sup _{1 \leq i \leq k}\left\{\frac{\mathbb{E}\left[\left|X_{i}\right|^{2+\alpha}\right]}{\sigma_{i}^{2+\alpha}}\left(\frac{\sigma_{i}}{B_{k}}\right)^{\alpha}\right\} \\
& \leq \limsup _{n \rightarrow \infty}|f|_{\text {Lip }} C_{\alpha, \lambda} \sup _{i \geq 1} \mathbb{E}\left[\left|X_{i}\right|^{2+\alpha}\right] \frac{1}{\log n} \sum_{k=1}^{n} \frac{1}{k\left(\inf _{i \geq 1} \sigma_{i}^{2}\right)\left(k \inf _{i \geq 1} \sigma_{i}^{2}\right)^{\frac{\alpha}{2}}} \\
& \quad=\frac{|f|_{\text {Lip }} C_{\alpha, \lambda} \sup _{i \geq 1} \mathbb{E}\left[\left|X_{i}\right|^{2+\alpha}\right]}{\left.\inf _{i \geq 1} \underline{\sigma}_{i}^{2}\right)^{1+\frac{\alpha}{2}}} \limsup _{n \rightarrow \infty} \frac{1}{\log n} \sum_{k=1}^{n} \frac{1}{k^{1+\frac{\alpha}{2}}} \\
& =0 .
\end{aligned}
$$

Therefore, equality (14) holds and the convergence rate is $O\left(\frac{1}{\log n}\right)$.

Theorem 4.5 Under the assumptions of Theorem 4.4, for $f \in C_{b, \mathrm{Lip}}(\mathbb{R})$ satisfying the following condition (16): for all $1 \leq l \leq k$,

there exist constants $\beta, c>0$ such that

$$
\left|\mathbb{E}\left[\left(f\left(W_{k}\right)-\mathbb{E}\left[f\left(W_{k}\right)\right]\right)\left(f\left(W_{l}\right)-\mathbb{E}\left[f\left(W_{l}\right)\right]\right)\right]\right| \leq c\left(\frac{l}{k}\right)^{\beta},
$$


we have

$$
\lim _{n \rightarrow \infty} \frac{1}{\log n} \sum_{k=1}^{n} \frac{f\left(W_{k}\right)}{k}=\widetilde{\mathbb{E}}[f(\xi)] \text { quasi-surely, }
$$

and the convergence rate is $o\left(\frac{1}{(\log n)^{\epsilon}}\right)$, for any $\epsilon \in(0,1 / 2)$.

Proof Under the condition (16), it follows from Theorem 4.2 that, for any $\epsilon \in(0,1 / 2)$,

$$
\frac{1}{\log n} \sum_{k=1}^{n} \frac{f\left(W_{k}\right)-\mathbb{E}\left[f\left(W_{k}\right)\right]}{k}=o\left(\frac{1}{(\log n)^{\epsilon}}\right) \text { quasi-surely. }
$$

On the other hand, from Theorem 4.4, we have

$$
\lim _{n \rightarrow \infty} \frac{1}{\log n} \sum_{k=1}^{n} \frac{\mathbb{E}\left[f\left(W_{k}\right)\right]}{k}-\widetilde{\mathbb{E}}[f(\xi)]=O\left(\frac{1}{\log n}\right)
$$

Consequently, we can get (17) and the convergence rate.

\section{Acknowledgements}

The authors would like to thank the editor and referees for their valuable comments.

\section{Funding}

This paper is supported by the National Natural Science Foundation of China (Grant Nos. 11601280 and 11871050) and the Natural Science Foundation of Shandong Province of China (Grant Nos. ZR2016AQ11 and ZR2016AQ13).

\section{Availability of data and materials}

Data sharing not applicable to this paper as no data sets were generated or analyzed during the current study.

\section{Competing interests}

The authors declare that they have no competing interests.

\section{Authors' contributions}

All authors contributed equally in writing this paper. All authors read and approved the final manuscript.

\section{Publisher's Note}

Springer Nature remains neutral with regard to jurisdictional claims in published maps and institutional affiliations.

Received: 26 November 2018 Accepted: 9 May 2019 Published online: 21 May 2019

\section{References}

1. Agahi, H., Mohammadpour, A., Mesiar, R., Ouyang, Y.: On a strong law of large numbers for momtone measures. Stat. Probab. Lett. 83, 1213-1218 (2013)

2. Chen, Z: Strong laws of large numbers for sub-linear expectations. Sci. China Math. 59(5), 945-954 (2016)

3. Chen, Z., Hu, C., Zong, G.: Strong laws of large numbers for sub-linear expectation without independence. Commun. Stat., Theory Methods 46(15), 7529-7545 (2017)

4. Chen, Z., Huang, H., Wu, P.: Extension of the strong law of large numbers for capacities. Math. Control Relat. Fields 9(1), 175-190 (2019)

5. Chen, Z., Wu, P., Li, B.: A strong law of large numbers for non-additive probabilities. Int. J. Approx. Reason. 54(3), 365-377 (2013)

6. De Cooman, G., Miranda, E.: Weak and strong laws of large numbers for coherent lower previsions. J. Stat. Plan. Inference 138, 2409-2432 (2008)

7. Denis, L., Hu, M., Peng, S.: Function spaces and capacity related to a sub-linear expectation: application to G-Brownian motion paths. Potential Anal. 34(2), 139-161 (2011)

8. Fazekas, I.: On a general approach to the strong laws of large numbers (2014). arXiv:1406.2883v1

9. Fazekas, I., Klesov, O.: A general approach to the strong laws of large numbers. Theory Probab. Appl. 45(3), 568-583 (2001)

10. Hu, S., Hu, M.: A general approach rate to the strong law of large numbers. Stat. Probab. Lett. 76(8), 843-851 (2006)

11. Longnecker, M., Serfling, R.J.: General moment and probability inequalities for the maximum partial sum. Acta Math. Acad. Sci. Hung. 30(1-2), 129-133 (1977)

12. Maccheroni, F., Marinacci, M.: A strong law of large number for capacities. Ann. Probab. 33, 1171-1178 (2005) 
13. Peng, S.: G-Expectation, G-Brownian motion and related stochastic calculus of Itô type. In: Stochastic Analysis and Applications, pp. 541-567. Springer, Berlin (2007)

14. Peng, S.: Survey on normal distributions, central limit theorem, Brownian motion and the related stochastic calculus under sub-linear expectations. Sci. China Ser. A, Math. 52(7), 1391-1411 (2009)

15. Peng, S.: Nonlinear expectations and stochastic calculus under uncertainty — with robust central limit theorem and G-Brownian motion (2010). arXiv:1002.4546v1

16. Song, Y.: Normal approximation by Stein's method under sublinear expectations (2017). arXiv:1711.05384v1

17. Sung, S.H., Hu, T., Volodin, A.: A note on the growth rate in the Fazekas-Klesov general law of large numbers and on the weak law of large numbers for tail series. Publ. Math. (Debr.) 73(1-2), 1-10 (2008)

18. Terán, P.: A law of large numbers for the possibilistic mean value. Fuzzy Sets Syst. 245, 116-124 (2014)

19. Tómács, T:: A general method to obtain the rate of convergence in the strong law of large numbers. Ann. Math. Inform. 34, 97-102 (2007)

20. Tómács, T., Líbor, Z.: A Hájek-Rényi type inequality and its applications. Ann. Math. Inform. 33, 141-149 (2006)

21. Zhang, L.: Rosenthal's inequalities for independent and negatively dependent random variables under sub-linear expectations with applications. Sci. China Math. 59(4), 751-768 (2016)

22. Zhang, L.: Strong limit theorems for extended independent and extended negatively dependent random variables under non-linear expectations (2016). arXiv:1608.00710v1

\section{Submit your manuscript to a SpringerOpen ${ }^{\circ}$ journal and benefit from:}

- Convenient online submission

Rigorous peer review

- Open access: articles freely available online

- High visibility within the field

- Retaining the copyright to your article

Submit your next manuscript at $\boldsymbol{\nabla}$ springeropen.com 